\title{
Experimental Testing of TCP/IP/Ethernet Communication for Automatic Control
}

\author{
Przemyslaw Plesowicz and Mieczyslaw Metzger \\ Faculty of Automatic Control, Electronics and Computer Science, \\ Silesian University of Technology, \\ ul. Akademicka 16, 44-100 Gliwice, Poland \\ \{przemyslaw.plesowicz, mieczyslaw.metzger\}@polsl.pl
}

\begin{abstract}
The TCP/IP/Ethernet protocol is considered not suitable for use in real-time control systems. It deals with a lack of time determinism, which characterizes fieldbuses. Nevertheless several corporations propose networking based on the TCP/IP/Ethernet even for control purposes with some modifications of the standard however. This paper examines possibility of application of the TCP/IP/Ethernet communication without modifications (introducing also Internet as one of tested cases) for feedback control purposes. Experimental investigations have been performed in four stages. In the beginning tests of network properties, including tests of transmission time and packet loss measurements have been performed. Three following stages show experimental testing of feedback control, when TCP/IP transmission occurs between PI controller and control plant. Three representative platforms have been chosen for testing: LabVIEW, RSLogix and Simatic. The main and original contribution presented in this paper is design and construction of three test stands as well as methodology of testing experiments. Standard control over analog channel has been also presented as comparison. The results of testing show acceptable performance of control via TCP/IP/Ethernet networking.
\end{abstract}

Keywords: TCP/IP communication testing, Ethernet TCP/IP, networks, network-based feedback control.

\section{Introduction}

Nowadays automation systems designed for industrial plants became complex and usually consist of many components such as instrumentation, software and networking. A growing need for advanced industrial networking techniques for complex applications in engineering and research results in more and more sophisticated technologies such as Profibus, Modbus, ControlNet, DeviceNet, CAN, FIP and many others. Mentioned standards have an important advantage over the widely used Ethernet standard - they are time-deterministic. Unfortunately, the application of fieldbuses has been limited due to very high cost of appropriate hardware and software and due to incompatibility of multivendor products. This situation has pushed engineers toward attempts to apply worldwide-used and in consequence inexpensive Ethernet standard. This is the reason, why appropriate 
testing techniques are crucial for such based on instrumentation, software and networks control systems.

Fundamentals of basic testing techniques for communication systems are well defined in recent publications over the last decade (see for example [1], [2], [3]). Although such methods are very convenient for complex communication systems in a general case, the specialised experimental tests dealing with development of laboratory stands seems to be more adequate for testing communication in automatic control. This paper presents such instrumentation and tests - designed, developed and carried out for testing TCP/IP/Ethernet communication in feedback control.

Remote data-acquisition and monitoring of non-critical plants can be achieved without deterministic characteristics, hence the TCP/IP/Ethernet can be used for remote monitoring without problems, with some requirements and modifications of the standard however - see for example [4], [5], [6]. The control theory proposes some analytical discussions of the problem of delays introduced by networking in control systems — see e.g. [7], [8], [9], [10].

This paper examines possibility of application of the TCP/IP/Ethernet communication without modifications (introducing also Internet as one of tested cases) for feedback control purposes. Experimental investigations have been performed in four stages. In the beginning, tests of network properties have been performed (including measurements of transmission time and packet loss). Three following stages show experimental testing of feedback control, in which TCP/IP transmission occurs between PI controller and control plant. Three representative platforms have been chosen for testing: LabVIEW (National Instruments), RSLogix (Rockwell-Allen-Bradley) and Simatic (Siemens). The hardware (PLC and distributed $\mathrm{I} / \mathrm{O})$ used during experiments was especially chosen, to represent solutions most popular in industry (Rockwell-Allen-Bradley, Siemens) and most popular in scientific research and education (National Instruments). The main and original contribution presented in this paper is design and construction of three test stands as well as methodology of testing experiments. As comparison, standard control over analog channel has been also presented. The results of testing show acceptable performance of control via TCP/IP/Ethernet networks.

\section{Motivation}

Using TCP/IP protocols, it is possible to build modular automatic control systems, where controllers are connected with plant using TCP/IP through SCADA (supervisory control and data acquisition) mediating software [11] (Fig. 1). Modular design of plant-controller system allows easy testing of various automatic control algorithms. Using more sophisticated mediating software, it is possible to connect more clients to the plant: remote automatic controllers, own SCADA systems, historical modules, databases and other applications using control/measurement data (Fig. 3). Usually however, such testing activities are preceded by simulation. Using simulator (Fig. 2) in place of real plant assures similar conditions during experiments, but also additionally repeatability of testing conditions. Using TCP/IP also in this case allows taking advantage of modular system design. Additionally, plant simulation for educational purposes provides protection against physical damage due to improper control of real (physical) plant. During tests presented in this paper, use of real 
(physical) plants was possible, taking however above presented statements in consideration, presented tests have been performed using virtual (simulated) plants.

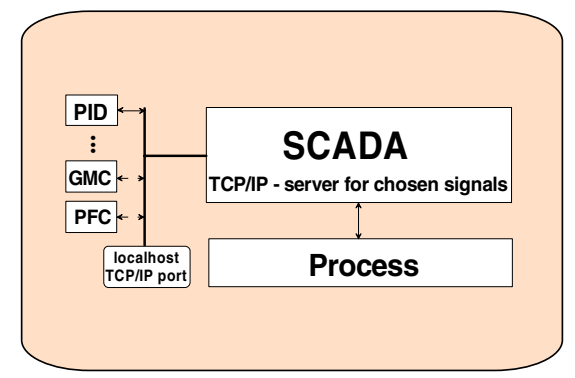

Fig. 1. Plant with SCADA software and .con trollers connected [11]

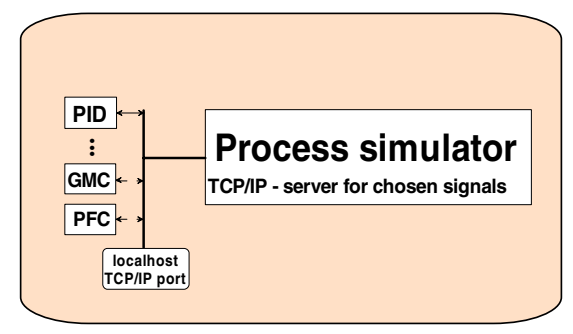

Fig. 2. Process (plant) simulator

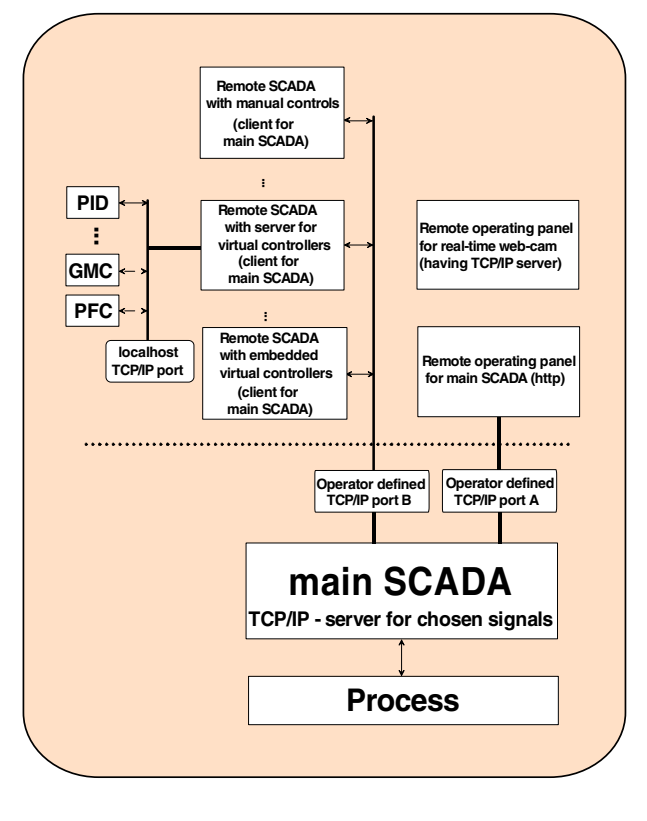

Fig. 3. Advanced architecture, allowing creation of individual SCADA systems [11]

\section{Tests of TCP/IP/Ethernet network Properties}

\subsection{Tests of Transmission Time}

One of the most important parameters describing the operation of computer network as a part of automatic control system is transmission delay. The purpose of these experiments was to measure the influence of software (operating system) and hardware on transport delay of TCP/IP/Ethernet-based local area network.

Materials and methods: The test environment consisted of two PCs, equipped with FastEthernet network cards, Ethernet switch, and crossed cable. All connections have been configured to $100 \mathrm{Mb} / \mathrm{s}$, Full Duplex. During the experiments operation of Microsoft Windows XP, QNX v.6.2 and Linux (kernel 2.4) have been tested.

Experiments: To minimize measurement errors, tests have been conducted with minimal load of tested systems (absolute minimal number of process running). Round Trip Time has been measured (using ping tool) for the following parameters:

- $\quad$ packet size: $64[\mathrm{~B}]$,

- $\quad$ packet sending frequency: $1,10,100,1000\left[s^{-1}\right]$,

- connection via: Ethernet switch ("switch"), crossed cable ("cross"),

- operating system: Windows XP - “win”, Linux - “lin”, QNX 6.2 - “qnx”. 


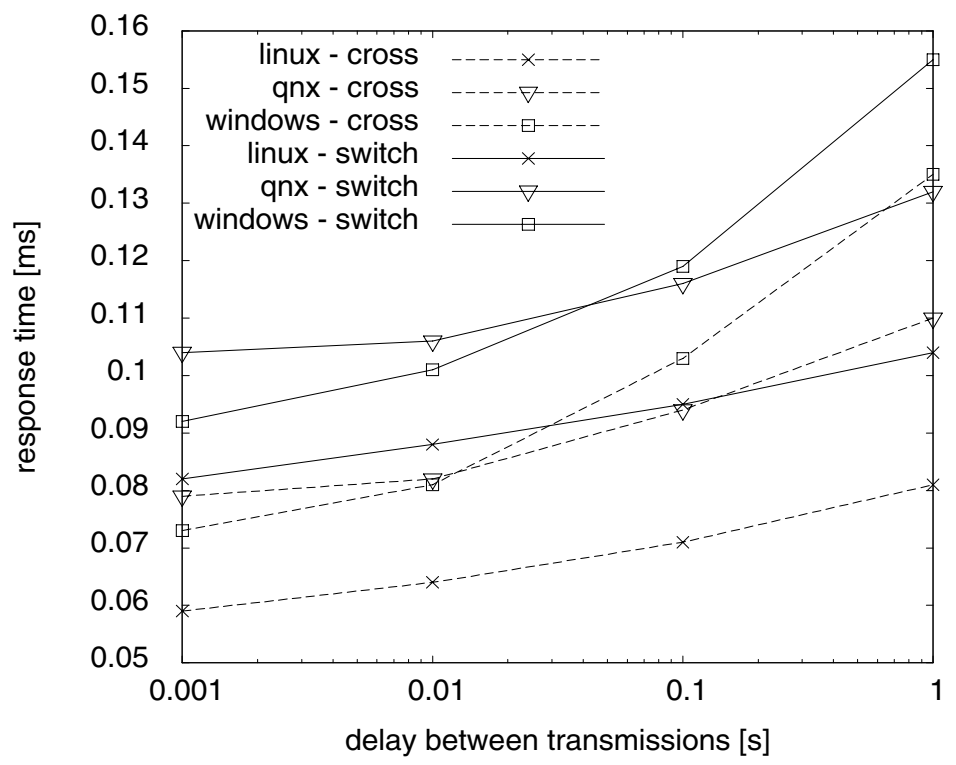

Fig. 4. Comparison of mean response time of Linux, $Q N X$, Windows XP, operating systems

Results: Table 1 shows comparison of measured RTTs for different operating systems and different connections. Considering the results it is possible to conclude that:

- transmission time changes in obvious way for different operation system,

- $\quad$ opposed to anticipations, QNX operating system advertised as real-time $O S$ required the longest time to respond. Analyzing time deviations, it is possible to state that QNX does not present higher determinism than other operating systems (in area of network transmission),

- for higher packet sending rate, transmission time is even $40 \% \div 50 \%$ shorter compared to slower sending rates. This phenomenon occurs in both cases ("cross" and "switch"), and is caused probably by operating system allocating more resources for more resource demanding processes (or not removing the process code from cache memory),

- the delay of $15 \div 25 \mu \mathrm{s}$ - introduced by Ethernet switch is clearly visible,

- $\quad$ in case of unmodified version of Linux, removing unnecessary processes and using closed network enables shortening bidirectional transmission time to $<0.15[\mathrm{~ms}]$, where deviation is lower than $0.05[\mathrm{~ms}]$,

\subsection{Packet Loss Measurement}

Second important factor influencing operation of transmission channel is the number of errors occurring. Knowledge of error ratio and transmission time (without acknowledgments) allows calculation of effective transmission time when retransmission occurs. 
Table 1. Comparison of Round Trip Time — various operating systems

\begin{tabular}{|c|c|c|c|c|c|c|}
\hline \multirow{2}{*}{$\begin{array}{r}\text { connection } \\
\text { cross }\end{array}$} & \multirow{2}{*}{$\begin{array}{l}\begin{array}{l}\text { sending speed } \\
\text { [packet/s] }\end{array} \\
1\end{array}$} & \multirow{2}{*}{$\begin{array}{r}\text { operating } \\
\text { system }\end{array}$} & \multicolumn{3}{|c|}{ time (min/mean/max/dev) } & \multirow[b]{2}{*}{013} \\
\hline & & & 0.073 & 0.081 & 0.109 & \\
\hline & & qnx & 0.100 & 0.110 & 0.159 & 0.016 \\
\hline & & win & 0.098 & 0.135 & 0.147 & 0.008 \\
\hline & 10 & $\operatorname{lin}$ & 0.062 & 0.071 & 0.093 & 0.011 \\
\hline & & qnx & 0.086 & 0.094 & 0.156 & 0.011 \\
\hline & & win & 0.089 & 0.103 & 0.119 & 0.008 \\
\hline & 100 & $\operatorname{lin}$ & 0.060 & 0.064 & 0.081 & 0.010 \\
\hline & & qnx & 0.075 & 0.082 & 0.102 & 0.009 \\
\hline & & win & 0.071 & 0.081 & 0.106 & 0.010 \\
\hline & 1000 & $\operatorname{lin}$ & 0.057 & 0.059 & 0.093 & 0.009 \\
\hline & & qnx & 0.075 & 0.079 & 0.131 & 0.008 \\
\hline & & win & 0.070 & 0.073 & 0.164 & 0.013 \\
\hline \multirow[t]{12}{*}{ switch } & 1 & $\operatorname{lin}$ & 0.092 & 0.104 & 0.115 & 0.014 \\
\hline & & qnx & 0.120 & 0.132 & 0.167 & 0.015 \\
\hline & & win & 0.114 & 0.155 & 0.166 & 0.009 \\
\hline & 10 & lin & 0.089 & 0.095 & 0.110 & 0.009 \\
\hline & & qnx & 0.108 & 0.116 & 0.132 & 0.010 \\
\hline & & win & 0.107 & 0.119 & 0.137 & 0.015 \\
\hline & 100 & lin & 0.083 & 0.088 & 0.107 & 0.012 \\
\hline & & qnx & 0.099 & 0.106 & 0.128 & 0.005 \\
\hline & & win & 0.091 & 0.101 & 0.122 & 0.010 \\
\hline & 1000 & lin & 0.079 & 0.082 & 0.122 & 0.010 \\
\hline & & qnx & 0.099 & 0.104 & 0.167 & 0.006 \\
\hline & & win & 0.088 & 0.092 & 0.166 & 0.009 \\
\hline
\end{tabular}

Table 2. Comparison of transmission errors

\begin{tabular}{clrrrrrr}
\hline connection & $\begin{array}{l}\text { sending } \\
\text { [packet/s] }\end{array}$ & speed & \multicolumn{2}{l}{$\begin{array}{l}\text { packet number } \\
\text { (sent/lost) }\end{array}$} & & & \\
switch & $10^{3}$ & $10^{8}$ & 388 & 0.072 & 0.076 & 2.088 \\
& $10^{2}$ & $10^{7}$ & 39 & 0.072 & 0.084 & 0.864 \\
& $10^{1}$ & $10^{6}$ & 4 & 0.090 & 0.106 & 0.243 \\
cross & $10^{0}$ & $10^{5}$ & 1 & 0.126 & 0.137 & 0.261 \\
& $10^{3}$ & $10^{8}$ & 1 & 0.058 & 0.062 & 1.890 \\
& $10^{2}$ & $10^{7}$ & 0 & 0.058 & 0.070 & 0.857 \\
& $10^{1}$ & $10^{6}$ & 0 & 0.075 & 0.091 & 0.207 \\
& $10^{0}$ & $10^{5}$ & 0 & 0.111 & 0.122 & 0.216 \\
\hline
\end{tabular}

Materials and methods. To minimize measurement errors, tests have been conducted with minimal load of tested systems (absolute minimal number of process running). Number of lost packets (and Round Trip Time) has been measured (using ping tool) for the following parameters: 
- packet size: $36[B]$,

- packet number: 100 ,

- packet sending frequency: $1,10,100,1000\left[s^{-1}\right]$,

- connection via: Ethernet switch ( "switch"), crossed cable (“cross"),

Results and conclusions. Because of the test formula, it is necessary to remember, that single measurement consists of bidirectional packet transmission, so the real error ratio is equal approximately 0.5 of measured value. Analyzing the results, several phenomena are observable:

- Ethernet switch is main cause of packet loss - the ratio of packet lost when using switch compared to transmission using crossed cable is $\approx 400$ to 1 ,

- during switched packet transmission, probability of error (packet loss) is $\approx 2 * 10^{-6}(2 \mathrm{ppm})$, but probability of error in transmission using crossed should be considered as value under measurement accuracy.

- there is no observable influence of transmission speed on amount of errors in tested range $(1 \div 1000$ packets/s).

Additionally, result similar to shown in subsection (3.1) has been observed:

- using switch, the packet transmission time is approximately $15 \div 25 \mu \mathrm{s}$ longer, than in case of crossed cable,

Final conclusion is, that in real switch-based local area network expected value of packet loss is approximately $1 \div 10 \mathrm{ppm}$ on every switch. For higher packet sending rate, transmission time is even $40 \% \div 50 \%$ shorter compared to slower sending rates. This phenomenon occurs in both cases ("cross" and "switch"), and is caused probably by operating system allocating more resources for more resource demanding processes (or not removing the process code from cache memory). Occurrence of longer transmission time for lower packet sending frequency shows clearly, that it is not only TCP/IP/Ethernet what contributes to longer transmission delays. Longer and unpredictable delays are caused mainly by resource management strategy and timesharing (multitasking) procedures of operating system.

\section{Influence of Transmission Time on Control Quality (LabVIEW Platform)}

To evaluate applicability of TCP/IP/Ethernet based networks for automatic control purposes, several tests have been performed. During tests, influence of transmission delay introduced by computer network on control quality in automatic control system (Plant-Controller) has been measured.

The first-order plus dead-time (FOPDT) dynamics has been chosen as control plant. Most of process control plants have or can be approximated by this form of the model. Also PI controller has been chosen because its simplicity and popularity in industry. 


\subsection{Materials and Methods}

The test environment consisted of two PCs, equipped with FastEthernet network cards and AD/DA interface cards (National Instruments). The test applications used during experiments (virtual plant, virtual controller and TCP/IP networks) have been built using LabVIEW environment (National Instruments). Standard, unmodified TCP/IP/Ethernet has been used for signal transmission.

$L a b V I E W$ has been chosen, during the test software design process, because its position as de facto standard in area of automatic control test software.

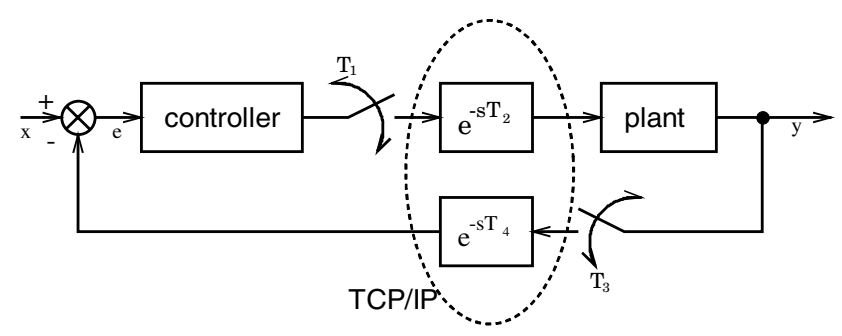

Fig. 5. PI controller connected via TCP/IP to FOPDT plant

When the controller and the plant are realized as real-time applications (in LabVIEW or LabVIEW-RT environments) the control system under consideration can be treated as a virtual control system. Such a way of simulations is very helpful for testing of control algorithms in conditions closest to the industrial reality. Additionally the virtual parts of the system can be replaced by corresponding commercial real-world components. In standard applications investigated controller and plant should be connected by industry standard signals (4-20 mA). Such a way of testing requires very expensive I/O hardware. The connection based on the TCP/IP can be low-cost alternative of automatic control systems testing. When values transmitted via digital channel are equal to values of the industry standard, the tested automation component can be easily used for connection with real-world component by industry standard.

The process model and controller have been realized as real-time simulator in the LabVIEW environment. A more detailed description of how to realize real-time simulation of the system (presented in Fig. 5) can be found in [12].

The term "virtual controller" seems to be well defined. The virtual controller must include at least all professional controller features, such as anti-reset windup action as well as bumpless switching between manual and automatic control. Without possibility of I/O connection, such a controller should be treated as only simulated controller (for simulation of control systems). For use as the virtual controller, this controller must include the connections with an I/O-PC-board or I/O modular system. For investigations presented here, the controller must include the 
TCP/IP socket as well. The PI controller has been built using the NI-LabVIEW environment.

\subsection{Experimental Tests}

Several experimental tests have been made, to discover possible differences between automatic control qualities. For a comparison, additional test without TCP/IP has been performed (communication using local variables).

Following connection channels have been tested:

- locally connected (in one computer, without TCP/IP) -"no-net"

- locally TCP/IP-connected (in one computer) - "localhost"

- TCP/IP-connected in intranet (local based network) - "local-net"

- TCP/IP-connected in Internet - "internet"

- TCP/IP-connected via network simulator -"netsim"

Following connection channels have been tested:

- $\quad$ time constant of the plant: $T_{1}=1[\mathrm{~s}] ; 20[\mathrm{~s}]$

- $\quad$ plant dead-time: $T_{0}=0 ; 0.25 T_{1} ; 0.5 T_{1}$

- $\quad$ process variable sampling time: $R T_{i}=0.05[\mathrm{~s}] ; 0.2[\mathrm{~s}]$

- $\quad$ selected pairs of controller gain and integration time: $\left(k R ; T_{i}\right)$

The data for charts has been collected with rate of 40 samples per second. Round Trip Time in tested intranet was measured: $\approx 0.3 \mathrm{~ms}$, and Internet: $\approx 35 \mathrm{~ms}$.

The Wide Area Network connection (Gliwice-Warsaw, Poland; $350 \mathrm{~km}$ distance) used in these experiments was low quality link - with high packet loss, and long maximal transmission time (up to $\approx 1500 \mathrm{~ms}$ ).

\subsection{Observations and Conclusions}

The results presented in Fig. 6,7 show the system response after step change of set point from $40 \%$ to $50 \%$.

Presented results show the satisfactory control quality in all cases except the Internet, however by making additional assumptions it is also possible to obtain satisfactory control quality using Internet. It is noticeable, that there are almost no differences present between the first three characteristics ("no-net", "localhost", "local-net"). Thus, it is possible, to make the following conclusion: for not too fast plants (time constant higher than 1s), TCP/IP based feedback loop in intranet offers control performance and quality very similar to the best performance available for discrete systems. This can be derived from the fact, that in most cases no difference between control over LAN and control in one computer (even without use of TCP protocol stack) is observed.

Problems with satisfactory control quality occurred, only in case of Internet connection (very low link quality) of controller and fast plant (time constant of $1 \mathrm{~s}$, Fig. 6). Having tested plant with longer time constant (20s and higher), almost no difference in control quality had been observed compared to local area network even when low quality WAN link had been used (see Fig.7). 


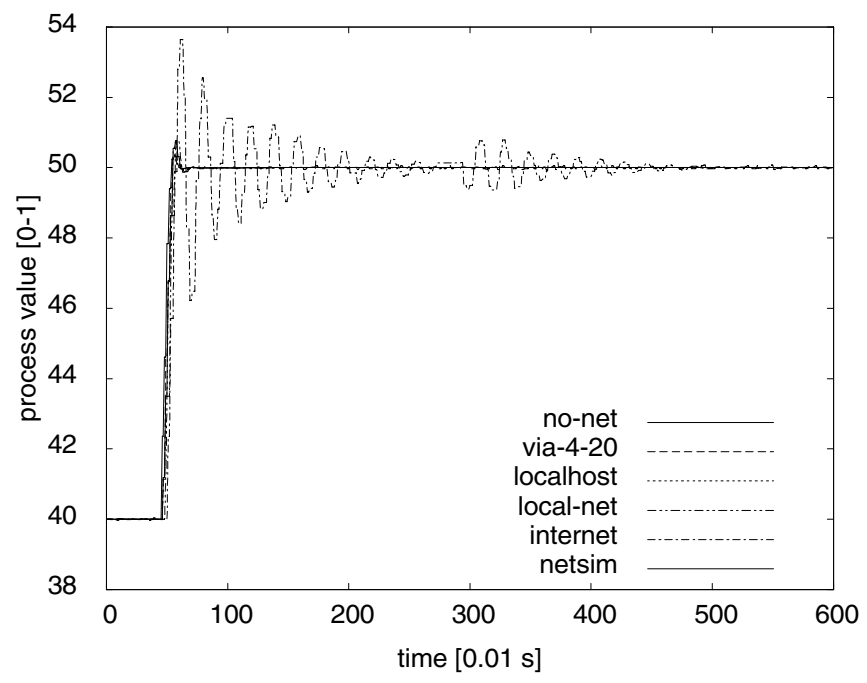

Fig. 6. Typical system response. Parameters: $k R=12.0 ; T_{i}=0.6[s] ; R T_{i}=0.05[s] ; T_{1}=1.0[s]$; $T_{0}=0$.

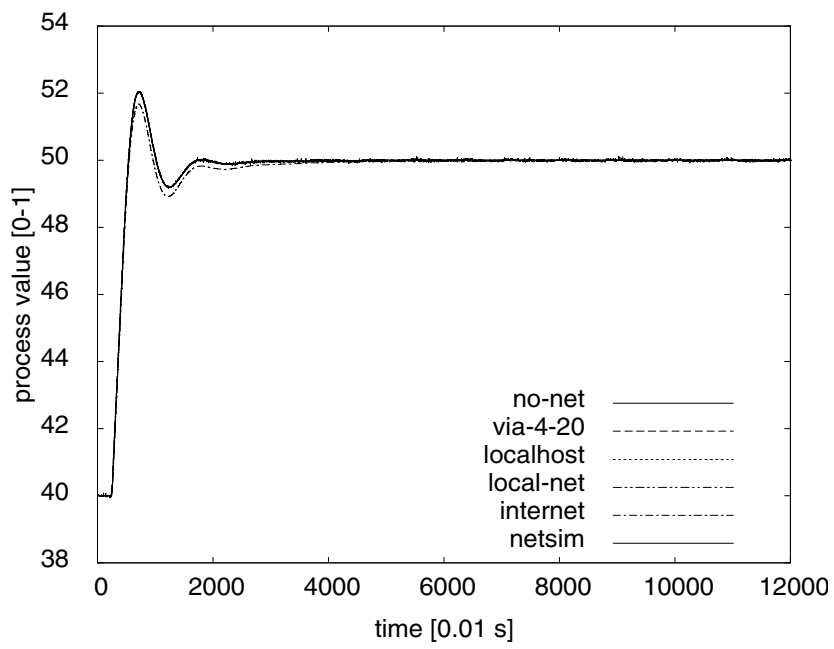

Fig. 7. Selected worst-case system response. Parameters: $k R=3.0 ; T_{i}=24[s] ; R T_{i}=0.05[s] ; T_{1}$ $=20.0[s] ; T_{0}=5.00[s]$.

\section{Influence of Transmission Time on Control Quality (Logix Platform)}

Use of Logix platform (Rockwell Automation) was caused by desire to test highestclass automation hardware and software widely used in industry, with communication 
channels based on $C I P-$ EtherNet/IP $-T C P / I P$ protocols and Ethernet devices. Results here presented, have also been initially signalized in [13].

\subsection{Materials and Methods}

A special laboratory stand has been developed for experimental testing. The test environment consisted of one PC, equipped with FastEthernet network card and AD/DA interface cards (National Instruments), programmable logic controller FlexLogix 5434 (Rockwell Automation), with Ethernet/IP communication module and Ethernet/IP-equipped distributed I/O - FlexIO. As OPC server RS-Linx has been used.

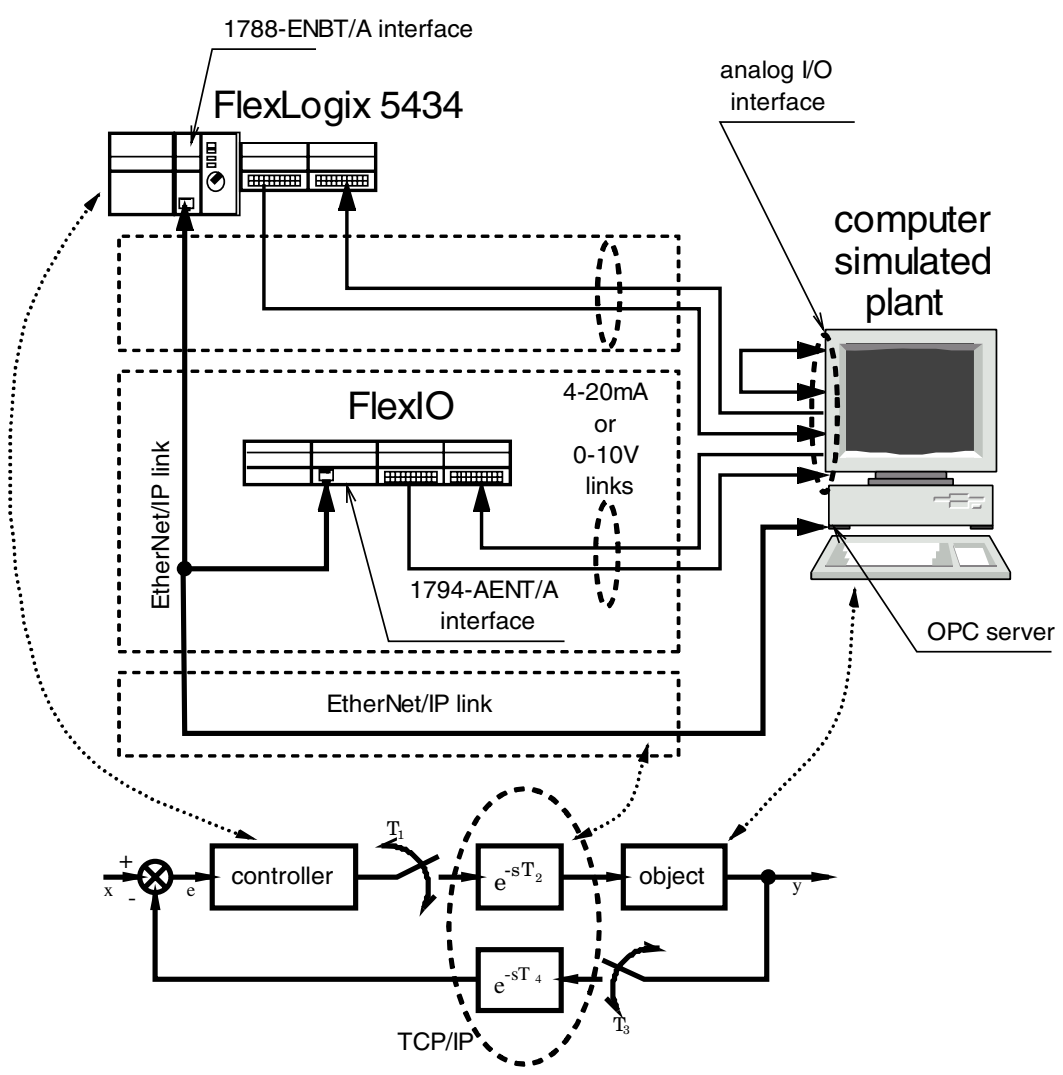

Fig. 8. Architecture of comparative test setup (Logix, EtherNet/IP) [13]

The applications used during testing experiments (plant simulator) have already been described in section (3.1), but additionally OPC communication routines have been added. For plant control, standard PID controller available in FlexLogix 5434 PLC has been used. 
Because of earlier made thesis, stating that speed instabilities of PLC's and computers has got significant influence on tests, special test environment has been prepared to eliminate this influence. This resulted in design presented in Fig. 8, in which signal transmission occurred via $0-10 \mathrm{~V}$, or EtherNet/IP+0-10V (to allow differential measurements). This solution allows also obtaining independence from AD/DA converters delay and other delays not associated with transmission. Thus, the tests have been performed in following setups:

- no tests with local connection (in one computer) have been made simulation of PLC's internal PID controller characteristics was not possible,

- $\quad$ plant and controller connected using 0-10V channel,

- $\quad$ plant and controller connected using EtherNet/IP and 0-10V channel,

- $\quad$ plant and controller connected using $O P C$ protocol based channel,

Results of tests presented in section 4 showed, that TCP/IP/Ethernet influence becomes significant only in case of short plant time constants, thus the experiments have been performed for all defined parameters with special attention to "bad" plant dynamics. In this case biggest differences between control responses can be expected.

Following preset values have been taken into consideration.

- $\quad$ plant time constant: $T_{1}=1[\mathrm{~s}]$

- $\quad$ plant dead-time: $T_{0}=0 ; 0.25 T_{1} ; 0.5 T_{1}$

- $\quad$ process variable sampling time: $R T_{i}=0.01[\mathrm{~s}]$

- $\quad$ selected pairs of regulator gain and integration time: $\left(k R ; T_{i}\right)$

- data for charts has been collected with rate of 100 samples per second,

- Round-Trip-Time in tested intranet was measured: $\approx 0.3 \mathrm{~ms}$.

\subsection{Results and Conclusions}

The responses, which are presented in Fig. 9, are the selected, worst-case system responses after step change of set point from 0 to 0.5 .

It should be noticed, that there are almost no differences present between the first two control responses ("0-10V", "EtherNet/IP+O-10V”). Thus, it is possible, to make following conclusion: for not too fast plants (plant time constant higher than 1s), Ethernet/IP based feedback via intranet offers control performance and quality very similar to the best performance available (" $0-10 \mathrm{~V}$ " — analog connection). In case of signal transmission using OPC protocol - even with the best parameters chosen - the control quality degrades significantly. This fact is probably caused by data exchange desynchronization in OPC server. Hence, it is possible to conclude, that OPC protocol server (in case of $R S$-linx) is not suitable as feedback transmission channel of systems with short time constant. It should be noted however, that this statement should be verified with experiment - the control quality degradation could have been caused by OPC server version of particular vendor (Rockwell AutomationAllen-Bradley).

As shown in this paper, even though Ethernet-TCP/IP network (and consequently Allen-Bradley's EtherNet/IP) is considered to be time-nondeterministic - it is possible to build automatic control system using this type of intranet. In local area 
networks (intranets), with Ethernet switch used for collision domains separation EtherNet/IP seems to be good communication medium for automatic control.

Presented experimental results show, that considering typical local network time delays and plant time constants, automatic control using EtherNet/IP channel is similar to the quality of system using analog channel. The use of $O P C$ protocol for control of plants having short time constants seems to be problematic however.

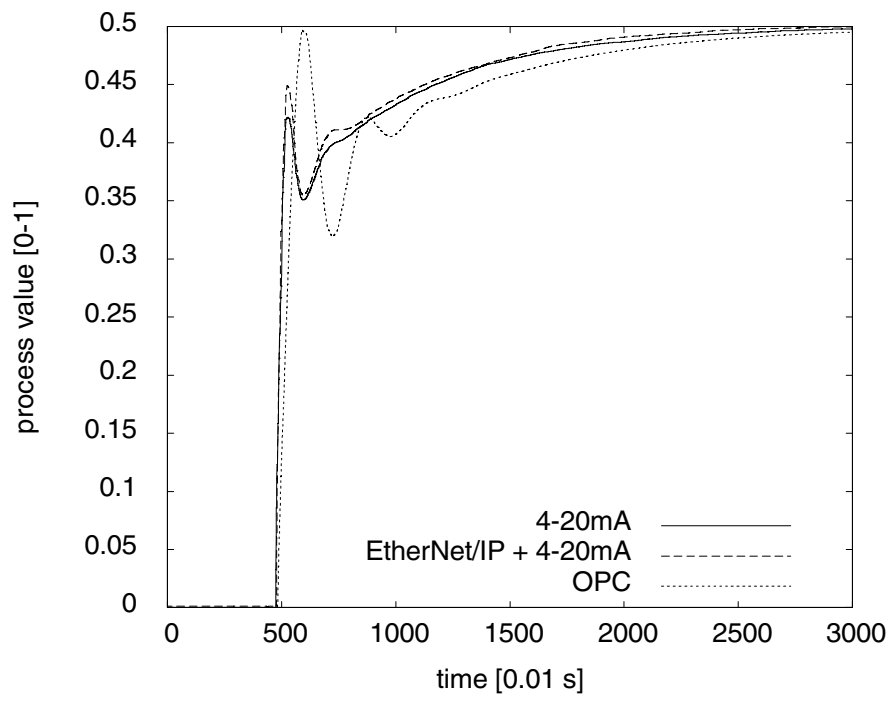

Fig. 9. Selected worst-case system response. Parameters: $k R=1.4 ; T_{i}=18[s] ; R T_{i}=0.01[s] ; T_{1}$ $=1.0[s] ; T_{0}=0.5[s]$.

During the tests interesting phenomena have been observed - large execution time instabilities of main program loop. In case of FlexLogix 5434, execution time (and its variation) of the same program was much bigger $(0.2-2.3 \mathrm{~ms})$ than transmission time in local area network $(0.07-0.15 \mathrm{~ms})$. This leads to conclusion, that often it is not computer network that causes control quality deterioration. Another fact has also been observable - due to low computational power of PLC hardware, their response time was much longer than PC with the same communication interfaces.

\section{Influence of Transmission Time on Control Quality in Simatic Platform}

In this section, results of Simatic (Siemens) hardware and software have been presented. Hardware and software of this manufacturer was chosen because of its popularity in European industry.

\subsection{Materials and Methods}

A special laboratory stand has been developed for experimental testing. The test environment consisted of one PC, equipped with FastEthernet network cards and 
AD/DA interface card (National Instruments Lab-PC+), Simatic S7-300 programmable logic controller (Siemens).

The test applications used during experiments (plant simulator) have already been described in section (3.1). Standard, unmodified TCP/IP/Ethernet was used for signal transmission. For plant control, standard PID controller available in Simatic S7-300 PLC had been used.

Because of earlier statement that speed instabilities of PLCs and computers has got significant influence on tests, special test environment has been prepared to eliminate this influence. This resulted in design presented in Fig. 10, in which signal transmission occurred via $0-10 \mathrm{~V}, T C P / I P+0-10 \mathrm{~V}$, and $T C P / I P-$ connected via proxy in industrial computer - for analog $(0-10 \mathrm{~V})$ digital conversion $(T C P / I P /$ Ethernet $)$.

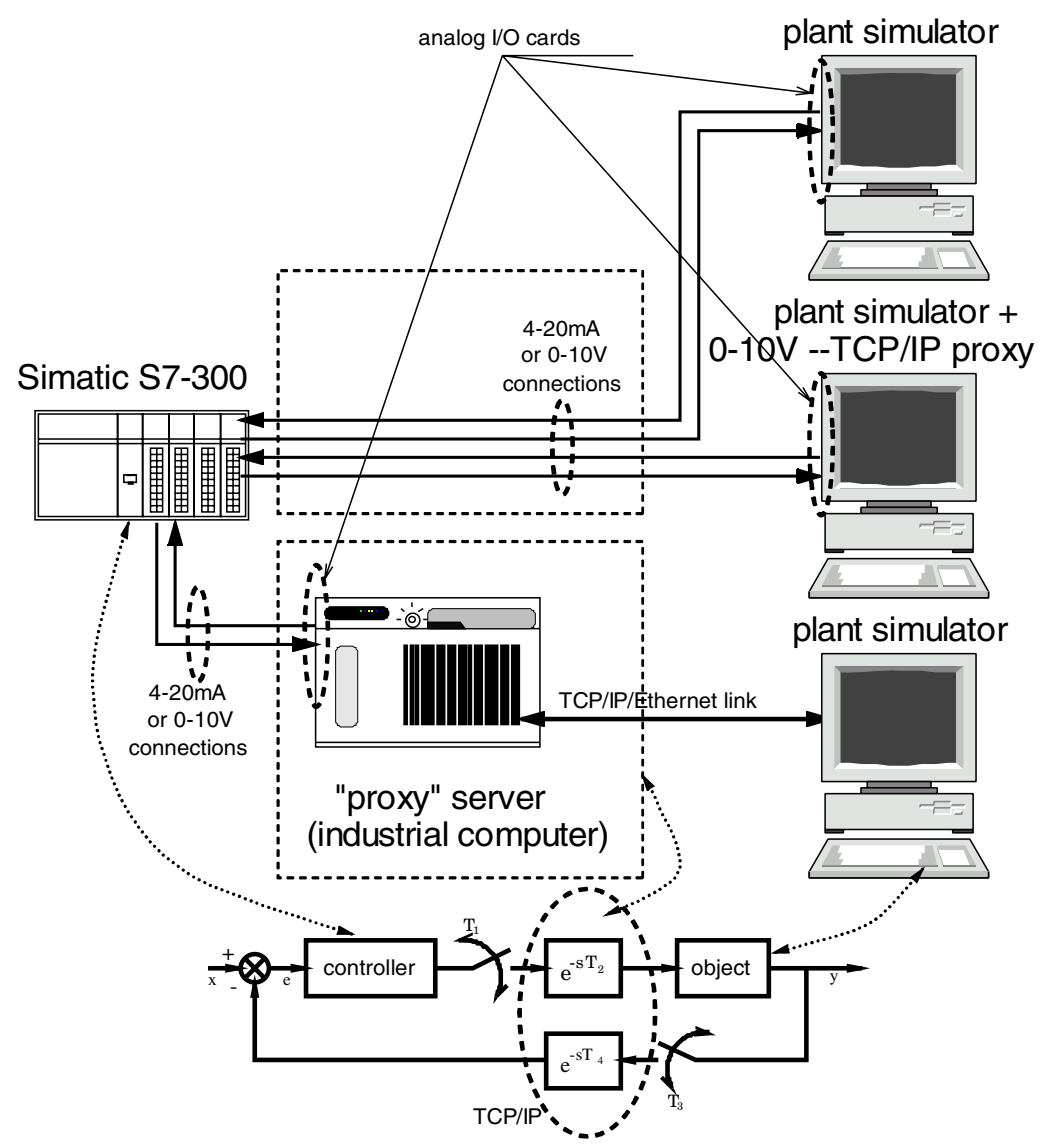

Fig. 10. Architecture of comparative test setup (Simatic)

This solution allows differential measurements and obtaining independence from AD/DA converters delay and other delays not associated with transmission. 
The tests have been performed in following setups (Fig. 10):

- no tests with local connection (in one computer) have been made simulation of PLC's internal PID controller characteristics was not possible,

- $\quad$ plant and controller connected using 0-10V channel ("localhost" in figure),

- plant and controller connected using $0-10 \mathrm{~V}$ and TCP/IP channel, in one operating system, using proxy ("proxy" in figure),

- $\quad$ plant and controller connected using $0-10 \mathrm{~V}$ and TCP/IP channel, in local area network, using proxy ( "intranet" in figure),

Because results of tests presented in section (3) showed, that TCP/IP/Ethernet influence becomes significant only in case of short plant time constants, the experiments have been performed for all defined parameters with special attention to "bad" plant dynamics (similar to those presented in section 3). In this case biggest differences between control responses can be expected.

Following preset values have been taken into consideration.

- $\quad$ plant time constant: $T_{1}=1[\mathrm{~s}]$

- $\quad$ plant dead-time: $T_{0}=0 ; 0.25 T_{1} ; 0.5 T_{1}$

- $\quad$ process variable sampling time: $R T_{i}=0.01[\mathrm{~s}]$

- $\quad$ selected pairs of regulator gain and integration time: $\left(k R ; T_{i}\right)$

- data for charts has been collected with rate of 100 samples per second,

\subsection{Results and Conclusions}

The result presented in Fig. 11 is the selected, worst-case system response after step change of set point from 0 to 0.5 .

It is noticeable, that control quality of system using $T C P / I P+0-10 \mathrm{~V}$ connection is worse than system using analog connection only $(0-10 \mathrm{~V})$.

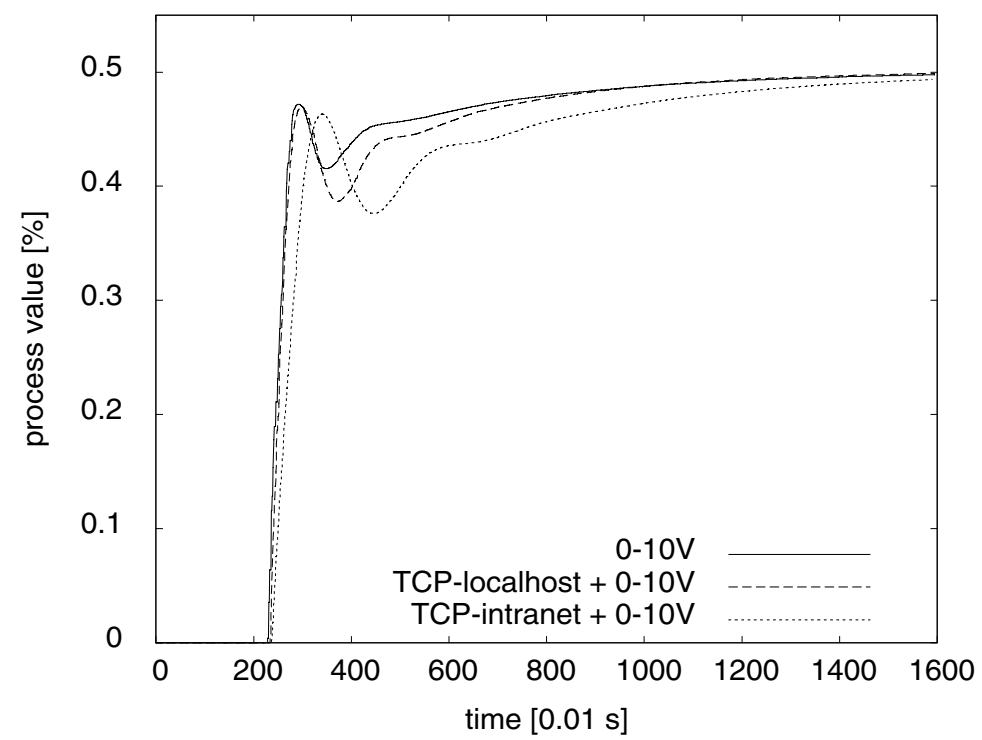

Fig. 11. Selected worst-case system response. Parameters: $\left.k R=1.4 ; T_{i}=3 s\right] ; R T_{i}=0.01[s] ; T_{1}$ $=1.0[s] ; T_{0}=0.5[s]$. 
Deterioration of control quality occurs not only because of transmission delays in computer network, but also due to delays and desynchronization of data exchange in proxying software.

Thus, the final conclusion is, that during designing of automatic control systems, unless inevitable, it is better to avoid excessive data passing.

\section{Concluding Remarks}

Currently the analog transmission is the most popular transmission in the industrial feedback loops. Although the networked communication is used in higher-level automatic control (monitoring, SCADA systems) such kind of transmission is too expensive for application in control loops. That is why an effort to apply the most popular Ethernet TCP/IP transmission is very promising. In the paper this kind of transmission has been tested experimentally. Opposed to majority of papers dealing with transmission in control and computer science this paper focuses rather on presenting method for testing of automatic control systems, and showing example test results. The main contribution presented in this paper is design and construction of three test stands as well as methodology of testing experiments. Each test stand consists of plant, communication channel and automatic controller.

Based on the experiments, it is possible, to make the following conclusion: for not too fast plants (time constant higher than 1s), TCP/IP based feedback loop in intranet offers control performance and quality very similar to the best performance available for discrete systems. It also is possible to control plants with longer time constant $(20 \mathrm{~s}$ and higher) via the Internet with almost no signs of control quality deterioration.

In general, presented results show promising perspectives not only for intranets, but also for Wide Area Networks (Internet) for plants with medium and long time constants.

Acknowledgments. This work has been supported by the Polish Ministry of Scientific Research and Information Technology.

\section{References}

1. Tretmans, J.: Test generation with inputs, outputs and repetitive quiescence. Software Concepts and Tools 17(3), 103-120 (1996)

2. Petrenko, A., Yevtushenko, N., Bochman, G.v., Dssouli, R.: Testing in context: framework and test derivation. Computer Communications 19, 1236-1249 (1996)

3. Grieskamp, W., Tillmann, N., Veanes, M.: Instrumenting scenarios in a model-driven development environment. Information and Software Technology 46, 1027-1036 (2004)

4. Flammini, A., Ferrari, P., Sisinni, E., Marioli, D., Taroni, A.: Sensor interfaces: from fieldbus to Ethernet and Internet. Sensors and Actuators A 101, 194-202 (2002)

5. Maciel, C.D., Ritter, C.M.: TCP/IP Networking in Process Control Plants. Computer Industrial Engineering, vol. 35(3-4), pp. 611-614

6. Vitturi, S.: On the Use of Ethernet at Low Level of Factory Communication System. Computer Standards \& Interfaces 23, 267-277 (2001)

7. Belle Isle, A.P.: Stability of Systems with Nonlinear Feedback Through Randomly TimeVarying Delays. IEEE Transactions On. Automatic Control AC-20(1), 67-75 (1975) 
8. Krtolica, R., Özgüner, Ü., Chan, H., Göktaş, H., Winkelman, J., Liubakka, M.: Stability of Linear Feedback Systems with Random Communication Delays. International Journal of Control 59(4), 925-953 (1994)

9. Lee, K.C., Lee, S.: Performance evaluation of switched Ethernet for real-time industrial communications. Computer Standards \& Interfaces 24, 411-423 (2002)

10. Decotignie, J-D.: Etherne-Based Real-Time and Industrial Communications. In: Proceedings of the IEEE, vol. 93(6), pp. 1102-1117 (2005)

11. Metzger, M.: Virtual controllers improve Internet-based experiments on semi-industrial pilot plants. In: Proceedings of the 16-th IFAC Triennal World Congress, CD, Elsevier, Amsterdam (2005)

12. Metzger, M.: Modelling and simulation of the sampled-data control of the nonlinear, continuous, distributed parameter plant. 15th IMACS World Congress, Wissenschaft und Technik Verlag, Berlin, Systems Engineering, vol. 5, pp. 161-166 (1997)

13. Plesowicz, P., Metzger, M.: Experimental Evaluation Of Ethernet/IP-Interconnected Control Systems. In: Proceedings of the IFAC Workshop on Programmable Devices and Systems (2006) 